\title{
Nice guys: Homozygocity for the TPH2 -703G/T (rs4570625) minor allele promotes low aggressiveness and low anxiety
}

\author{
Kariina Laas ${ }^{1}$, Evelyn Kiive ${ }^{2}$, Jarek Mäestu ${ }^{3}$, Mariliis Vaht ${ }^{1}$, Toomas Veidebaum ${ }^{4}$, Jaanus Harro ${ }^{1}$ \\ ${ }^{1}$ Division of Neuropsychopharmacology, Department of Psychology, University of Tartu, Tartu, Estonia \\ ${ }^{2}$ Division of Special Education, Department of Education, University of Tartu, Tartu, Estonia \\ ${ }^{3}$ Department of Exercise and Sports Sciences, University of Tartu, Tartu, Estonia \\ ${ }^{4}$ National Institute for Health Development, Tallinn, Estonia
}

\begin{abstract}
Background: Tryptophan hydroxylase (TPH) is the rate-limiting enzyme in the synthesis of serotonin. We examined whether the TPH2 polymorphism -703G/T (rs4570625) is associated with aggressiveness and impulsivity, and the prevalence of psychiatric disorders, in a populationrepresentative sample.

Methods: We used self and proxy reports on aggressive behaviour in the younger birth cohort of the longitudinal Estonian Children Personality, Behaviour and Health Study collected at age 25, and earlier collected impulsivity and related data of both ECPBHS cohorts.

Results: The TT homozygous males reported less aggressive behaviour in the Life History of Aggression interview at age 25. They also had significantly lower scores in Illinois Bully Scale peer reports, and less ADHD symptoms rated by teachers both at ages 9 and 15. The TT homozygotes of both sexes had the lowest Maladaptive Impulsivity at ages 18 and 25, and the highest Adaptive Impulsivity at age 25. The TT homozygotes also had low depressiveness and trait anxiety by age 25 , and the odds ratio for the prevalence of anxiety disorders was 9.38 for the G-allele carriers.

Limitations: The main limitation of the study is the naturally occurring low number of subjects with the TT genotype.

Conclusions: Subjects with the TPH2 rs4570625 TT genotype, especially males, exhibit less aggression and a favourable impulsivity profile, and develop anxiety disorders by young adulthood less often.
\end{abstract}




\section{Background}

Tryptophan hydroxylase (TPH) is the rate-limiting enzyme in the synthesis of serotonin. The role of the serotonergic system in regulating emotions, including aggressiveness is relatively well established (e.g., Bortolato et al., 2013; Lesch et al., 2012; Miczek et al., 2015). However, there is no simple explanation how alterations in serotonergic homeostasis lead to different types of psychopathology, or other- or self-directed aggressive behaviour. Therefore, additional information about the role of naturally existing variations in the serotonin system, including genetic variation, in emotion regulation and aggressive traits is crucial for understanding the underlying neurobiology.

There are two isoenzymes of TPH in humans, TPH1 and TPH2, the latter being predominantly expressed in the brain (Carkaci-Salli et al., 2011; Walther et al., 2003). Variations in genes encoding both TPH-s have been linked to emotion regulation and aggression-related traits (Bortolato et al., 2013; Waider et al., 2011), including the potentially functional rs4570625 SNP in the TPH2 gene that leads to $\mathrm{G}$ to $\mathrm{T}$ base substitution in the promoter region at position -703 . The functionality of this polymorphism still requires establishment: Two studies have found differences in gene expression in haplotypes containing the -703G/T (Chen et al., 2008; Lin et al., 2007), but Scheuch et al. (2007) found no G/T difference in promoter activity. Nevertheless, in studies on brain and behaviour, the T-allele of the $-703 \mathrm{G} / \mathrm{T}$ polymorphism has been described as a psychiatric risk allele as it is linked to biased amygdala responsiveness (Brown et al., 2005; Canli et al., 2005) and is overrepresented in cluster B and C personality disorders, as well as in both affective and anxiety disorders with a sample of patients with personality disorders (Gutknecht et al., 2007). The meta-analysis by Gao et al. (2012) found T-allele carriers at greater risk of affective disorders, and one report (Mandelli et al., 2012) has suggested higher sensitivity to stressful life events in this genotype. In some contrast, Gutknecht et al. (2007) linked the haplotype containing rs4570625 Gallele to higher TPQ Harm Avoidance and NEO Neuroticism in healthy adults, and Reuter et al. (2007a) found lower harm avoidance in TT homozygotes. The G-allele has been found transmitted more often to the children affected by ADHD (Walitza et al., 2005) and OCD (Mössner et al., 2006); GG homozygotes, both controls and ADHD patients, had altered prefrontal function during response inhibition task (Baehne et al., 2009). Furthermore, increased frequency of the Gallele was associated with suicide attempts in patients with major depression (Yoon and Kim, 2009). Perez-Rodriguez et al. (2010) found evidence of the involvement of $T P H 2$ in aggressiveness 
but they tested haplotypes that did not contain rs4570625. TPH2 -703G/T SNP may have important effect on susceptibility to suicidal behaviour in major depression.

In case of the TPH2 G/T polymorphism, for practical reasons, carriers of the low frequency Tallele have mostly been compared with the GG genotype. However, in our recent study (Lehto et al., 2015) the population-derived sample was large enough to allow the comparison of all genotypes separately. We found that the TT homozygotes differed significantly from other genotype groups: Specifically, TPH2 G-allele homozygotes and GT heterozygotes had similar personality profiles while the TT homozygotes had significantly lower Neuroticism, and higher Extraversion and Conscientiousness.

Given the important role of serotonergic function in emotion regulation and aggressiveness, and the findings on TPH2 described above, we sought to test how the variation in TPH2 rs 4570625 affects aggressive behaviour and related traits, and prevalence of mood, anxiety and alcohol use disorders in a population-representative sample of young adults.

\section{Method}

\subsection{Sample}

This study was carried out on the Estonian sample of the European Youth Heart Study (1998/99), which was subsequently incorporated into the longitudinal Estonian Children Personality Behaviour and Health Study (ECPBHS). The EYHS sample of the ECPBHS consists of two birth cohorts. Data on aggressive and antisocial behaviour, bullying and victimisation are currently available for the younger cohort only. Hence, most of the present analysis is focused on data of this cohort, but relevant measures of both cohorts were subject to analysis if available. The rationale and procedure of sample formation have been described elsewhere in detail (Harro et al., 2001; Tomson et al., 2011). The total number of subjects in the first wave in 1998/99 was 1176; 583 in the younger cohort $\left(\mathrm{M}_{\mathrm{Age}}=9.6 \pm 0.5\right)$ and 593 in the older cohort $\left(\mathrm{M}_{\mathrm{Age}}=15.6 \pm 0.6\right)$. The follow-up studies for the younger cohort took place in $2004\left(\mathrm{n}=483, \mathrm{M}_{\mathrm{Age}}=15.3 \pm 0.5\right), 2007$ $\left(n=454, M_{\text {Age }}=18.3 \pm 0.5\right)$ and $2014\left(n=440, M_{\text {Age }}=25.3 \pm 0.5\right)$; for the older cohort, the follow-ups were in $2001\left(n=449\right.$, including 62 additional subjects, $\left.M_{\text {Age }}=18.4 \pm 0.9\right)$ and $2008(n=541$, $\mathrm{M}_{\mathrm{Age}}=24.7 \pm 0.7$ ). The number of subjects with valid genotype and psychometric data is given in 
Table 1. All the subjects were of Caucasian origin. The study was approved by the Tartu University Ethics Review Committee on Human Research.

\subsection{Measures}

\subsubsection{Illinois Bully Scale (IBS)}

Illinois Bully Scale is an 18-item scale with three subscales, Bully, Fight, and Victim, assessing the frequency of bullying behaviour, fighting, and victimization by peers (Espelage and Holt, 2001). We used the conventional self-report and a version of the scale adopted to be filled in by classmates where we asked subjects to rate their peers (peer reports, mostly two raters per person; each rater also reported on two classmates as a rule). In both cases, we asked subjects to recall the times in primary school assessing the frequency of listed behaviours in 5-point scale ranging from "never" to "very often". In case of peer ratings, subjects were shown the list of classmates and were asked to select the first one or two peers they remembered well. For this reason, the number of subjects for whom proxy reports were obtained is larger than the number of participants in this particular study wave. The list was narrowed gradually as reports were obtained, but when a subject could not make a decision, he/she was permitted to pick someone from the list of already rated peers. Averaged scores were used in case of more than one rating per subject.

\subsubsection{ADHD symptoms by teacher reports}

ADHD symptoms were assessed by teachers as described in Kiive et al. (2010) using the Hyperactivity Scale (Af Klinteberg and Oreland, 1995). The data of both birth cohorts for ages 15 and 18 were combined, while for age 9, data were available for the younger birth cohort only (Table 1).

\subsubsection{Impulsivity self-reports}

Participants filled in the Adaptive and Maladaptive Impulsivity Scale (Laas et al., 2010; Paaver et al., 2008;) with subscales measuring Fast decision making and Excitement seeking (functional or 
adaptive impulsivity), and Disinhibition and Thoughtlessness (dysfunctional or maladaptive impulsivity). The data of both birth cohorts collected at ages 18 and 25 were combined (Table 1).

\subsubsection{Psychiatric interview at age 25}

Psychiatric assessment based on DSM-IV was carried out in both birth cohorts at age 25 (Table 1) by experienced clinical psychologists using the Mini-International Neuropsychiatric Interview (M.I.N.I.5.0.0; Sheehan et al., 1998; Estonian version: Shlik et al., 1999). We used lifetime prevalence of disorders in the analysis.

\subsubsection{Life History of Aggression interview}

The Life History of Aggression interview (LHA, Coccaro et al., 1997) was carried out in the younger birth cohort immediately after the M.I.N.I. interview to assess dimensions of aggression (Table 1). Items were scored only for the history of actual behaviour. LHA has three subscales: Aggression (temper tantrums, verbal aggression, indirect aggression, non-specific fighting, and physical assault against people); Consequences/Antisocial Behaviour (school disciplinary problems, problems with supervisors, antisocial behaviour not resulting in police involvement, and antisocial behaviour involving the police); and Self-Directed Aggression (self-injurious behaviour, and suicide attempts). Each item was rated on a 5-point scale, ranging from $0=$ No events to $5=$ More events than can be counted.

\begin{tabular}{|c|c|c|c|c|c|c|c|}
\hline \multirow{2}{*}{$\begin{array}{l}\text { Scale } \\
\text { Age }\end{array}$} & \multicolumn{4}{|c|}{ Younger cohort } & \multicolumn{3}{|c|}{ Older cohort } \\
\hline & $9 y$ & $15 \mathrm{y}$ & $18 \mathrm{y}$ & $25 \mathrm{y}$ & $15 \mathrm{y}$ & $18 \mathrm{y}$ & $25 \mathrm{y}$ \\
\hline M.I.N.I. psychiatric interview & - & - & - & 430 & - & - & 501 \\
\hline Life History of Aggression interview & - & - & - & 427 & - & - & - \\
\hline State-Trait Anxiety Inventory (State/Trait) & - & $449 /-$ & $445 / 439$ & $423 / 422$ & - & - & $475 / 477$ \\
\hline Montgomery-Åsberg & - & - & 421 & 425 & - & 437 & 538 \\
\hline \multicolumn{8}{|l|}{ Depression Rating Scale } \\
\hline Impulsivity & - & & 453 & 427 & - & 332 & 510 \\
\hline ADHD teacher's report & - & 401 & 224 & - & 580 & 310 & - \\
\hline Illinois Bully Scale self-report & - & - & - & 427 & - & - & - \\
\hline Illinois Bully Scale peer report & - & - & - & 507 & - & - & - \\
\hline
\end{tabular}




\subsubsection{TPH2 rs4570625 genotyping}

Genomic DNA was extracted from whole blood samples using Qiagen QIAamp® DNA Blood Midi Kit. Genotyping for TPH2 G-703T (rs4570625) was performed as described in Lehto et al. (2015) with the Applied Biosystems ViiA ${ }^{\text {TM }} 7$ Real-Time PCR System using the TaqMan® PreDesigned SNP Genotyping Assay with Solis BioDyne 5x HOT FIREPol@ Probe qPCR Mix Plus (ROX). All DNA samples were successfully genotyped. The genotype frequencies were in HardyWeinberg equilibrium. The distribution of TPH2 genotype (Table 2) was in Hardy-Weinberg equilibrium and did not differ between birth cohorts (Fisher's Exact Test $p=0.546$ ) and. The frequency of the minor T-allele was $21.8 \%$.

Table 2

Distribution of TPH2 rs4570625 genotype in both birth cohorts.

\begin{tabular}{lllll}
\hline Subsample & GG & GT & TT & Total \\
\hline Younger cohort & $356(61.4 \%)$ & $203(35.0 \%)$ & $21(3.6 \%)$ & $580(100 \%)$ \\
Older cohort & $393(60.1 \%)$ & $229(35.0 \%)$ & $32(4.9 \%)$ & $654(100 \%)$ \\
Total & $749(60.7 \%)$ & $432(35.0 \%)$ & $53(4.3 \%)$ & $1234(100 \%)$ \\
\hline
\end{tabular}

\subsection{Statistical analysis}

Subjects were analyzed by the $T P H 2$ genotype groups. Due to genotype distribution (see Table 2), the group sizes were too unequal to rely on parametric statistical tests like analysis of variance, so we have reported the results based on nonparametric tests. (ANOVA-s were, however, conducted, and the results were similar to the outcome of nonparametric tests.) We have used the following nonparametric tests for the scores of Illinois Bully Scale, LHA interview, ADHD report, impulsiveness and depressiveness: Kruskal-Wallis (KW) test for comparing three or more groups, Mann-Whitney (MW) $U$-test for two-group comparison, and interaction terms by adjusted rank transform (ART) test by Leys and Schumann (2010). The results for KW and MW $U$-tests are presented as raw p-values, and for the ART test as Fstatistic, raw p-value and $\eta^{2}$ as a measure of effect size. The tables contain main statistics for comparison of G-allele carriers with the TT genotype, and results for all three genotype groups are presented for illustrative purposes and justification of combining all G-allele carriers against the TT genotype. Statistical analysis of $T P H 2$ genotype distribution by lifetime presence of affective and anxiety disorders was performed with Fisher's exact test; odds ratios (OR) with confidence intervals (CI) were calculated as a 
measure of effect size. Contrasts were calculated for significant model effects. Calculations were made with SPSS.16. Whiskers indicate $95 \%$ confidence intervals. It was impossible to compute regression models with multiple predictors due to low number of TT homozygotes and missing data of different variables. The analysis was based on a priori hypotheses, so we have reported results as not corrected for multiple comparisons; a comment on implications of that is included in Discussion.

\section{Results}

\section{1. $\mathrm{TPH} 2$ genotype and aggressiveness in school by the Illinois Bully Scale}

A main effect of TPH2 genotype was found: peers had reported less victimization for the subjects with TT genotype (Table 3), the effect originating from males. In addition, male TT homozygotes had lower Fight scores (Fig. 1 and Table 3). Genotype $\times$ Sex interaction effect was significant only in case of the Fight subscale where male G-allele carriers scored significantly higher as compared to the TT males and all females (Fig. 1B, nonparametric ART test, $F(1,506)=9.74, p=0.002$, $\eta^{2}=0.019$ ). We found no statistically significant genotype differences in the IBS self-reports although similar tendencies were present.

\subsection{TPH2 genotype and aggressiveness by the LHA interview}

Male TT homozygotes scored much lower in the LHA interview (Table 4, Fig. 2), both in Aggression and Antisocial Behaviour subscales. Further analysis revealed that items in the Aggression subscale yielding genotype differences in males were Non-specific fighting (MW $U$ Test, G-allele carriers vs. TT genotype, $p=0.039)$, Verbal aggression $(p=0.031)$ and Indirect aggression $(p=0.049)$, but not Temper tantrums $(p=0.297)$ and Physical assault against people $(p=0.316)$. In the Antisocial Behaviour subscale, only School disciplinary problems individually yielded genotype differences in males $(p=0.033)$. There was no significant association of TPH2 genotype with Self-directed aggression (SDA) $\left(\chi^{2}=1.75, \mathrm{p}=0.186\right)$; however there were no TT subjects with self-directed aggressive behaviour $(\mathrm{G}$-allele + no SDA=367, G-allele + SDA=46, TT + no $\mathrm{SDA}=14, \mathrm{TT}+\mathrm{SDA}=0$ ). No genotype differences in the LHA score were found in females. 
Table 3

TPH2 effects on aggressiveness (the IBS subscales) by peer reports: group means and standard errors, and Mann-Whitney $U$-Test $p$-value. Bold - significant difference between G-allele carriers and the TT genotype.

\begin{tabular}{|c|c|c|c|}
\hline IBS subscales & $\begin{array}{l}\text { TPH2 main effect } \\
n_{\mathrm{G}+}=489, n_{\mathrm{TT}}=18\end{array}$ & $\begin{array}{l}\text { TPH2 Males } \\
n_{\mathrm{G}+}=229, n_{\mathrm{TT}}=8\end{array}$ & $\begin{array}{l}\text { TPH2 Females } \\
n_{\mathrm{G}+}=260, n_{\mathrm{TT}}=10\end{array}$ \\
\hline Victim & $\begin{array}{l}\mathrm{M}_{\mathrm{G}+}=7.46 \pm 0.15 \\
\mathrm{M}_{\mathrm{TT}}=5.14 \pm 0.79 \\
p=0.001\end{array}$ & $\begin{array}{l}M_{G+}=8.02 \pm 0.21 \\
M_{\mathrm{TT}}=4.90 \pm 1.15 \\
p=0.002\end{array}$ & $\begin{array}{l}\mathrm{M}_{\mathrm{G}+}=6.96 \pm 0.21 \\
\mathrm{M}_{\mathrm{TT}}=5.33 \pm 1.07 \\
p=0.096\end{array}$ \\
\hline Bully & $\begin{array}{l}\mathrm{M}_{\mathrm{G}+}=14.91 \pm 0.31 \\
\mathrm{M}_{\mathrm{TT}}=13.84 \pm 1.61 \\
p=0.211\end{array}$ & $\begin{array}{l}\mathrm{M}_{\mathrm{G}+}=16.69 \mid \pm 0.49 \\
\mathrm{M}_{\mathrm{TT}}=12.20 \pm 2.60 \\
p=0.069\end{array}$ & $\begin{array}{l}\mathrm{M}_{\mathrm{G}}=13.34 \pm 0.37 \\
\mathrm{M}_{\mathrm{TT}}=15.15 \pm 1.89 \\
p=0.792\end{array}$ \\
\hline Fight & $\begin{array}{l}\mathrm{M}_{\mathrm{G}_{+}}=7.93 \pm 0.18 \\
\mathrm{M}_{\mathrm{TT}}=6.39 \pm 0.91 \\
p=0.082\end{array}$ & $\begin{array}{l}M_{G+}=9.83 \pm 0.28 \\
M_{T T}=6.02 \pm 1.52 \\
p=0.003\end{array}$ & $\begin{array}{l}\mathrm{M}_{\mathrm{G}_{+}}=6.26 \pm 0.16 \\
\mathrm{M}_{\mathrm{TT}}=6.68 \pm 0.80 \\
p=0.638\end{array}$ \\
\hline
\end{tabular}

A Males

Females

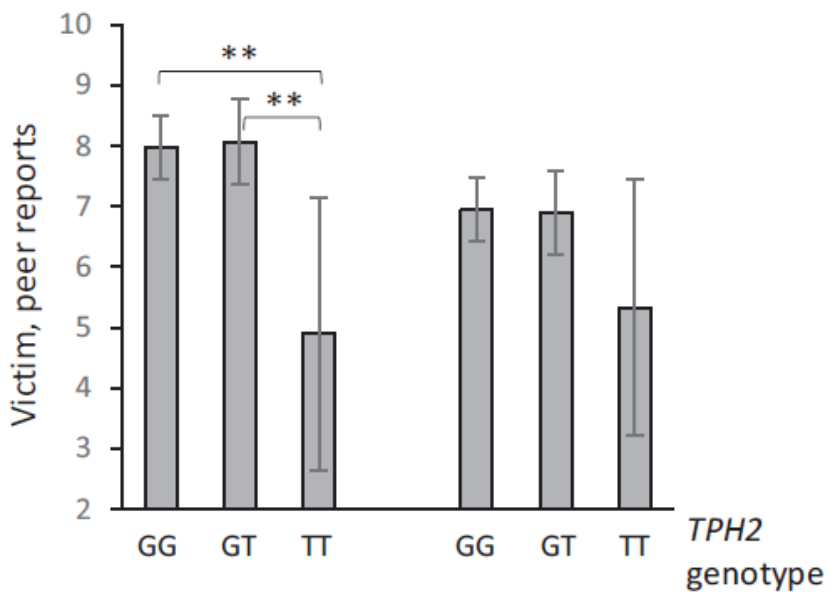

B

Males

Females

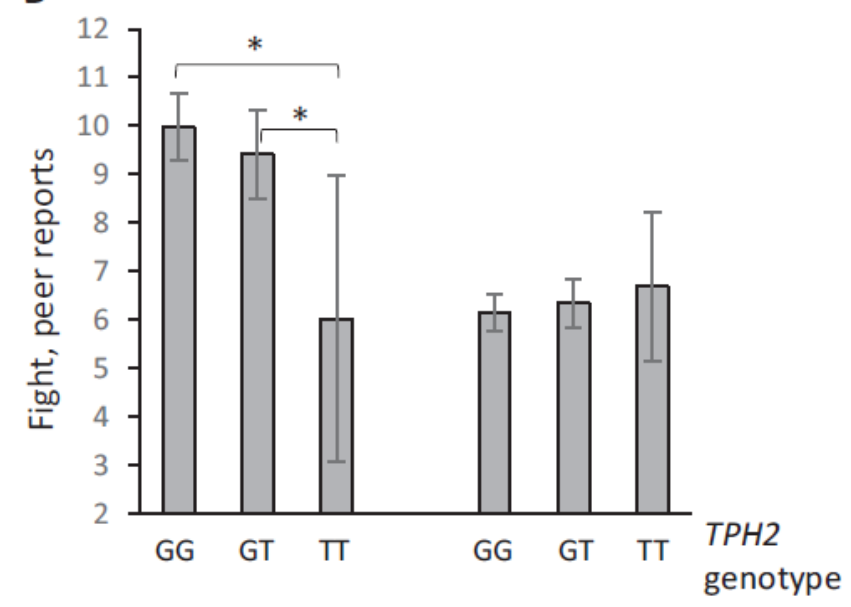

Fig. 1. Examples of TPH2 association with the Illinois Bully Scale peer reports, $n=507$. (A) Subscale Victim, TPH 2 main effect by Kruskal-Wallis Test $p=0.003$, males $p=0.007$, females $p=0.222$. (B) Subscale Fight, TPH2 main effect $p=0.186$, males $p=0.007$, females $p=0.886 .{ }^{*}-p<0.05,{ }^{* *}-p<0.01$. 
Table 4

TPH2 effects on aggressiveness (LHA subscales) in males and females: Group means and standard errors, and statistical assessment by the Mann-Whitney $U$-Test. Bold significant difference between G-allele carriers and the TT genotype.

\begin{tabular}{llll}
\hline \multirow{2}{*}{ LHA subscales } & TPH2 main effect & TPH2 Males & $\begin{array}{l}\text { TPH2 Females } \\
n_{\mathrm{G}+}=413, n_{\mathrm{TT}}=14\end{array}$ \\
& $n_{\mathrm{G}+}=182, n_{\mathrm{TT}}=7$ & $n_{\mathrm{TT}}=7$ \\
\hline \multirow{2}{*}{ Aggression } & $\mathrm{M}_{\mathrm{G}+}=6.17 \pm 0.22$ & $\mathbf{M}_{\mathbf{G}+}=\mathbf{7 . 7 0} \pm \mathbf{0 . 3 6}$ & $\mathrm{M}_{\mathrm{G}+}=4.95 \pm 0.26$ \\
& $\mathrm{M}_{\mathrm{TT}}=3.93 \pm 1.22$ & $\mathbf{M}_{\mathbf{T T}}=\mathbf{3 . 4 3} \pm \mathbf{1 . 8 2}$ & $\mathrm{M}_{\mathrm{TT}}=4.43 \pm 1.51$ \\
& $p=0.055$ & $\boldsymbol{p = 0 . 0 1 6}$ & $p=0.626$ \\
Antisocial & $\mathrm{M}_{\mathrm{G}+}=2.12 \pm 0.14$ & $\mathbf{M}_{\mathbf{G}+}=\mathbf{3 . 7 9} \pm \mathbf{0 . 2 4}$ & $\mathrm{M}_{\mathrm{G}+}=0.81 \pm 0.10$ \\
Behaviour & $\mathrm{M}_{\mathrm{TT}}=1.36 \pm 0.75$ & $\mathbf{M}_{\mathbf{T T}}=\mathbf{1 . 4 3} \pm \mathbf{1 . 2 3}$ & $\mathrm{M}_{\mathrm{TT}}=1.29 \pm 0.54$ \\
& $p=0.413$ & $\boldsymbol{p = 0 . 0 4 2}$ & $p=0.622$ \\
LHA Total & $\mathrm{M}_{\mathrm{G}+}=8.58 \pm 0.33$ & $\mathbf{M}_{\mathbf{G}+}=\mathbf{1 1 . 6 4} \pm \mathbf{0 . 5 5}$ & $\mathrm{M}_{\mathrm{G}+}=6.17 \pm 0.33$ \\
& $\mathrm{M}_{\mathrm{TT}}=5.29 \pm 1.80$ & $\mathbf{M}_{\mathbf{T T}}=\mathbf{4 . 8 6} \pm \mathbf{2 . 8 1}$ & $\mathrm{M}_{\mathrm{TT}}=5.71 \pm 1.87$ \\
& $p=0.051$ & $\boldsymbol{p = 0 . 0 0 9}$ & $p=0.559$ \\
\hline
\end{tabular}

Males

Females

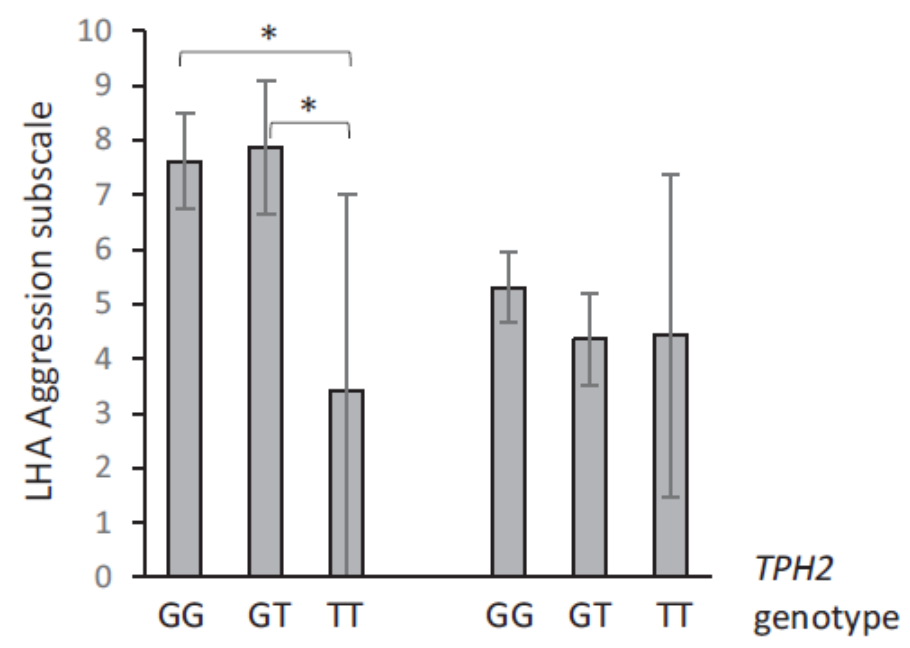

Fig. 2. TPH2 effect on Life History of Aggression Interview total scores, $n=427$. TPH2 main effect by Kruskal-Wallis Test, $p=0.058$, males $p=0.034$, females $p=0.289$. ${ }^{*}-p<$ 0.05 .

\subsection{TPH2 genotype, impulsiveness and ADHD symptoms}

$T P H 2$ genotype was associated with both impulsiveness and hyperactivity symptoms. For these analyses data of both birth cohorts were available. TPH2 TT homozygotes had lower Maladaptive impulsivity (MI) and higher Adaptive impulsivity (AI) at both ages 18 and 25 (Fig. 3A and B). At age 18, differences were significant in the whole sample (Fig. 3A), and in females analyzed separately (MI: KW test $p=0.002, \mathrm{M}_{\mathrm{GG}}=33.8 \pm 0.5 \mathrm{M}_{\mathrm{GT}}=34.1 \pm 0.6 \mathrm{M}_{\mathrm{TT}}=30.3 \pm 1.8$; AI: KW test $p=0.036, \mathrm{M}_{\mathrm{GG}}=37.1 \pm 0.5 \mathrm{M}_{\mathrm{GT}}=36.4 \pm 0.6 \mathrm{M}_{\mathrm{TT}}=38.4 \pm 1.9$ ), while at age 25 , genotype effects emerged only if males and females were combined. 

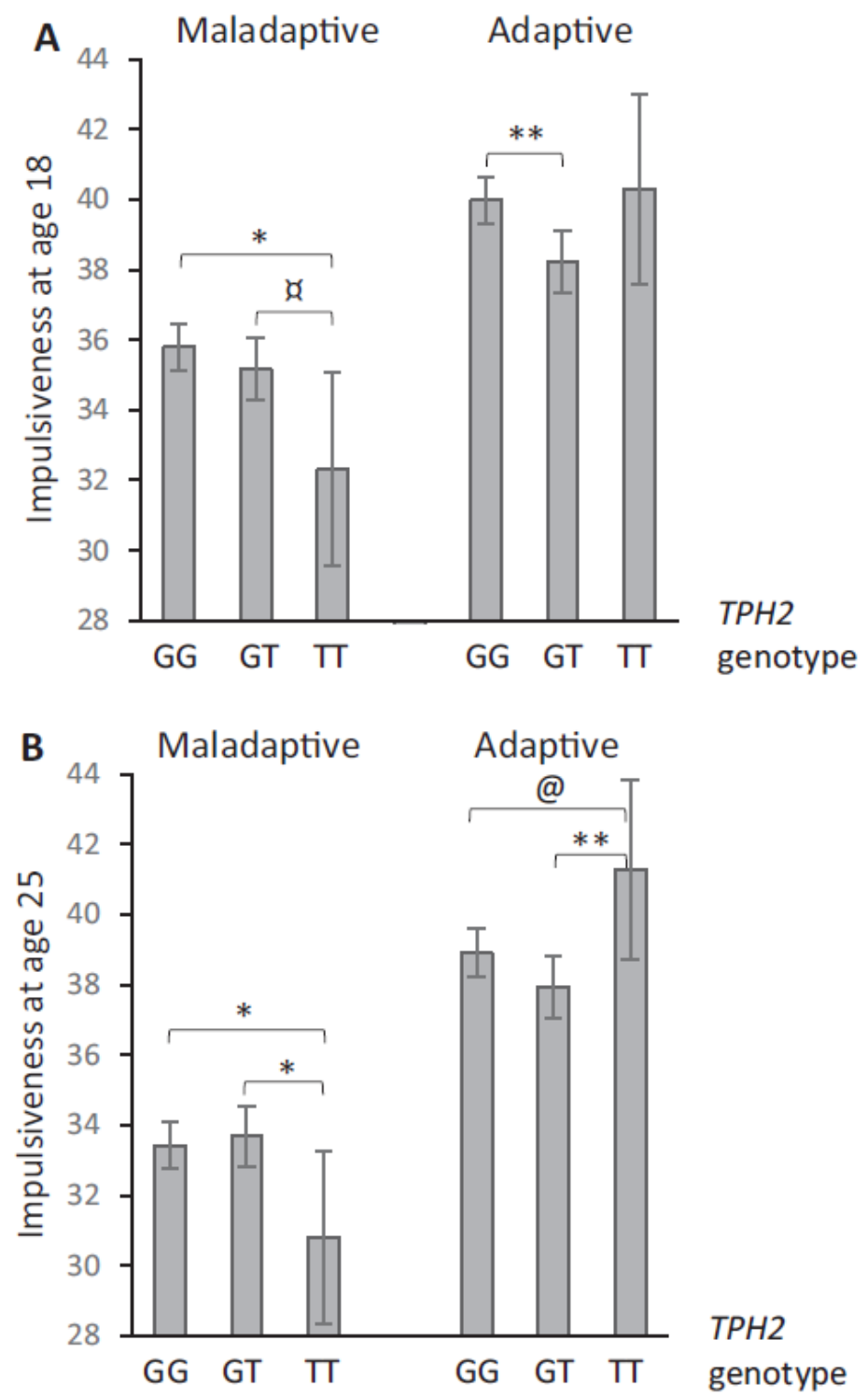

Fig. 3. TPH2 genotype association with impulsiveness at ages 18 and 25. (A) Impulsiveness at age 18 : MI, $p=0.082$; TT vs. G-allele $p=0.068$; $\mathrm{AI}, p=0.008$, TT vs. Gallele $p=0.646$. (B) Impulsiveness at age 25: MI, $p=0.094$; TT vs. G-allele $p=0.043$; AI, $p=0.035$; TT vs. G-allele $p=0.061$. @ $p=0.078$, $\not p=0.053$, ${ }^{*} p<0.05$, ${ }^{* *} p<0.01$, difference from indicated group.

TPH2 effect on teacher-rated ADHD symptoms was observable at younger age only. TPH $2 \times$ Sex interaction effect was present at the age of 9: Male TT heterozygotes had less hyperactivity symptoms while in females the trend was to the opposite (Fig. 4; data available only for the younger birth cohort; ART test G-allele vs. TT $\left.F(1486)=6.54, p=0.011, \eta^{2}=0.013\right)$. This was the case for both motor restlessness and concentration difficulties while analyzed separately (not 
shown). At ages 15 and 18, similar but statistically non-significant trends were detected, and there were no genotype differences in teacher-rated aggressive behaviour at any age.

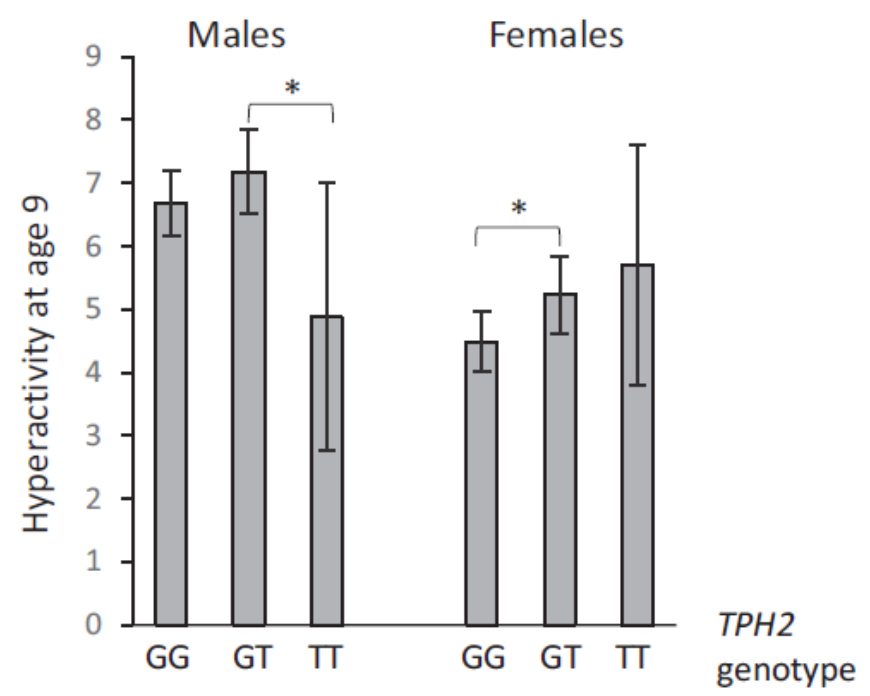

Fig. 4. TPH $2 \times$ Sex interaction effect on hyperactivity symptoms at age 9; ART test, $F(2$, 484) $=3.34, p=0.036, \eta^{2}=0.014$. ${ }^{*} p<0.05$.

\subsection{TPH2 genotype, and anxiety and affective disorders}

Only one subject with the TT genotype of the TPH2 rs4570625 polymorphism had met the criteria for anxiety disorders by age 25 so the TT homozygotes were 9 times less likely to have an anxiety disorder compared to G-allele carriers (Fig. 5; Fisher's Exact Test for G-allele vs. TT genotype, $p=0.003$, OR for G-allele 9.38, 95\% CI (1.28-68.8)). There was no difference in genotype distribution for mood and substance use disorders analyzed separately (Fisher's Exact Test $p=0.271$ and $p=0.440$, respectively). However, if combining the anxiety and mood disorders the TT subjects had significantly less affective/anxiety disorders (G-allele vs. TT genotype $p=0.018$, OR for Gallele 2.57, 95\% CI=1.13-5.88; G+/no disorder=581, G+/disorder=308, TT/no disorder=34, TT/disorder=7). These findings were consistent with genotypic differences in both depressiveness and anxiety self-reports at age 25: TT homozygotes had lower depressiveness as measured with MADRS $\left(\mathrm{M}_{\mathrm{G}+}=7.41 \pm 0.19, \mathrm{M}_{\mathrm{TT}}=5.24 \pm 0.88\right.$; MW $U$-Test $\left.\mathrm{p}=0.015\right)$ and lower scores of both Trait $\left(\mathrm{M}_{\mathrm{G}+}=38.8 \pm 0.3, \mathrm{M}_{\mathrm{TT}}=35.5 \pm 1.6 ; p=0.011\right)$ and State anxiety $\left(\mathrm{M}_{\mathrm{G}+}=31.3 \pm 0.3, \mathrm{M}_{\mathrm{TT}}=29.3 \pm\right.$ $1.4 ; p=0.045$ ). The differences were however not statistically significant at earlier ages (data not shown). While the differences at the age of 25 appeared similar in both sexes, the genotype effect 


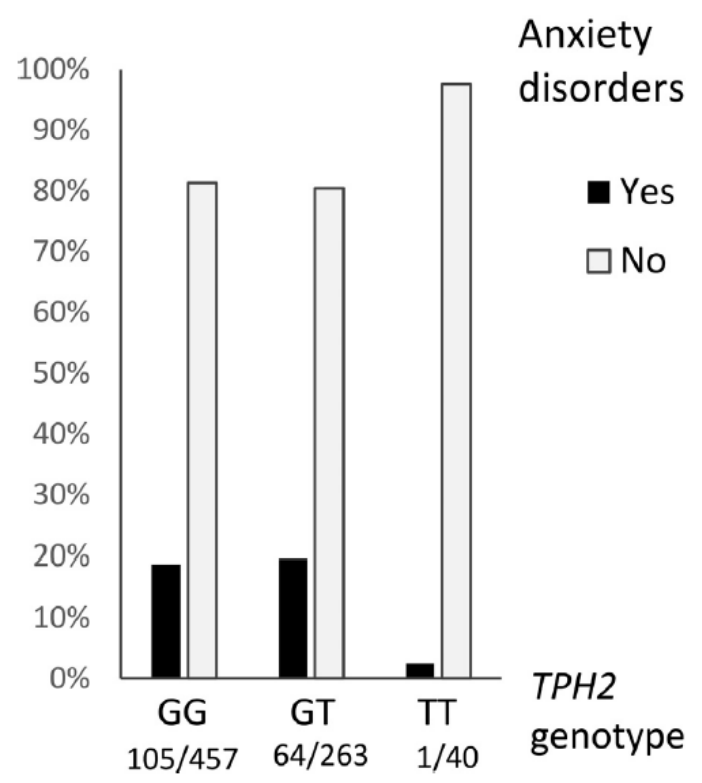

Fig. 5. TPH2 genotype and anxiety disorders by age 25. Fisher's Exact test for three genotype groups, $p=0.011$; G-allele vs. TT genotype $p=0.003$; OR for G-allele $9.38,95 \%$ CI (1.28-68.8).

on depressiveness and trait anxiety reached statistical significance only in males (depressiveness in males $\mathrm{M}_{\mathrm{G}+}=6.92 \pm 0.28, \mathrm{M}_{\mathrm{TT}}=4.48 \pm 1.18 ; p=0.040$, and females $\mathrm{M}_{\mathrm{G}+}=7.78 \pm 0.26, \mathrm{M}_{\mathrm{TT}}=6.05$ $\pm 1.30 ; \mathrm{p}=0.201$; trait anxiety in males $\mathrm{M}_{\mathrm{G}+}=37.2 \pm 0.4, \mathrm{M}_{\mathrm{TT}}=33.9 \pm 1.9 ; p=0.033$, and females $\left.\mathrm{M}_{\mathrm{G}+}=40.0 \pm 0.5, \mathrm{M}_{\mathrm{TT}}=37.3 \pm 2.5 ; p=0.171\right)$.

\subsection{TPH2 genotype effects on anxiety and depressiveness in alcohol use disorder}

Interestingly, the $T P H 2$ genotype effect on state anxiety and on depressiveness scores at age 25 were more pronounced in subjects who had by that age experienced alcohol use disorder (Fig. 6A and B). Thus, statistically significant genotype $\times$ diagnosis interaction effects were found (state anxiety, $F(2852)=5.53, p=0.004, \eta^{2}=0.013$; depressiveness, $\left.F(2910)=4.77, p=0.009, \eta^{2}=0.010\right)$. The $T P H 2$ effect on trait anxiety was similar but less clear (ART test $F(2852)=2.47, p=0.086$, $\eta^{2}=0.006$, in AUD group only KW test $p=0.005$ ). Nine males had the TT genotype and a record of alcohol use disorder, and the interactions in males were also statistically significant; as only two females had the TT genotype and history of alcohol use disorder the interaction was not separately analyzed. 

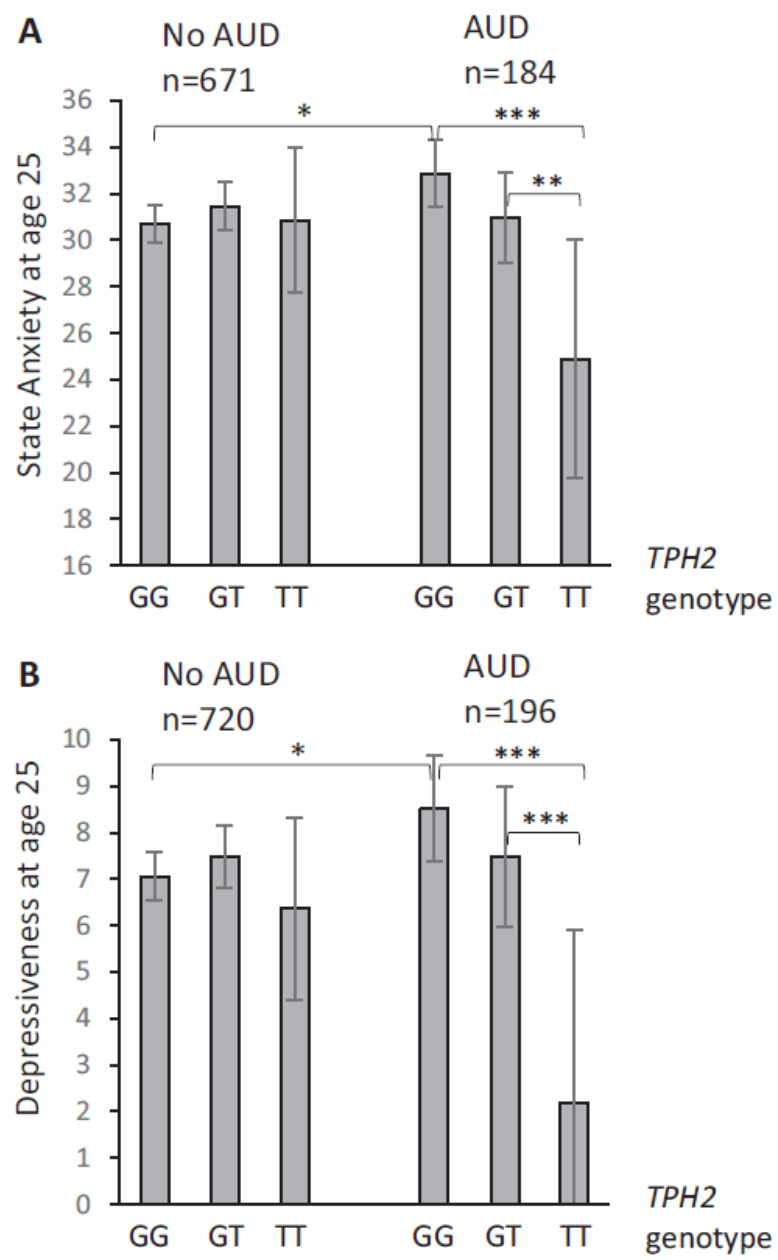

Fig. 6. TPH2 genotype effect on state anxiety and MADRS depressiveness dependent on the history of alcohol use disorder. (A) State Anxiety, ART test $F(2852)=5.53, p=0.004$, $\eta^{2}=0.013$. In AUD group only, KW test $p=0.003$. (B) MADRS, ART test $F(2910)=4.77$, $p=0.009, \eta^{2}=0.010$. In AUD group only, KW test $p=0.001$. ${ }^{*} p<0.05,{ }^{* *} p<0.01,{ }^{* *} p<$ 0.001 , difference from indicated group.

\section{Discussion}

Subjects with the low-frequency TT genotype of the TPH2 rs4570625 polymorphism differed significantly in various affect-related behaviours and traits by self- and proxy-reports and by psychiatric interviews. They had less aggressiveness, victimization, ADHD symptoms, impulsiveness, depressiveness and anxiety, and were less likely to correspond to diagnostic criteria of anxiety disorders as compared to G-allele carriers. The differences were more prominent in males, especially with regard to aggressive traits in childhood and in young adulthood.

Previous findings on the $T P H 2-703 \mathrm{G} / \mathrm{T}$ polymorphism have not been consistent with regard to which allele has been associated with the outcomes considered favourable. Either allele has been reported as the risk allele for different psychiatric disorders or disadvantages (e.g., Gao et al., 2012; 
Gutknecht et al., 2007; Mössner et al., 2006; Walitza et al., 2005; Yoon and Kim, 2009). Owing to the lower prevalence of the Tallele, the T-allele homozygotes and GT heterozygotes have been combined in these studies. Here using a sample highly representative of a population we could observe that while some differences were found between GG homozygotes and GT heterozygotes, these were minor, but the TT homozygotes differed with regard to many measures, suggestive of their better mental health. The neurobiological implications of this polymorphism are not entirely clear but the T-allele has been suggested to relate to hyperfunction of tryptophan hydroxylase (Chen et al., 2008; Lin et al., 2007). If this were true, serotonin levels could be higher particularly in the TT homozygotes, and this could well be associated with low aggressiveness, anxiety and depressiveness. Animal studies (Kriegebaum et al., 2010) have demonstrated that alterations in TPH2 function and synthesis of 5-HT are subject to potent compensatory mechanisms, and this could theoretically explain the minor effect of a single T-allele.

Certain limitations need be considered. Until replicated in other samples, one should remain cautious about interpretations of the present findings: While the sample has its merits in representativeness to population and in data collection at distinct ages, the main limitation of the study is the naturally occurring low number of subjects with the TT genotype. This has also prevented any more stratified analysis: We could not compute regression models with multiple predictors due to low number of TT homozygotes or to examine gene $\times$ environment interactions to check if the T-homozygotes react differently to environmental adversities as the serotonergic system, in which $T P H 2$ is a key player, has been shown to be environment-sensitive (e.g., Waider et al., 2011). Other components of the serotonin system could moderate the association: Chen et al. (2008) found that $-703 \mathrm{G} / \mathrm{T}$ exerts synergic effects with another upstream SNP $-473 \mathrm{~A} / \mathrm{T}$, and is in strong linkage disequilibrium with other SNP-s (Gutknecht et al., 2007; Walitza et al., 2005) that could have a role in emotion regulation; furthermore, TPH2 has additive effects with the 5HTT (Canli et al., 2008; Herrmann et al., 2007; Lehto et al., 2015), but all this can be examined together with the $T P H 2-703 \mathrm{G} / \mathrm{T}$ TT homozygocity only in very large samples. Finally, we have not corrected the results for multiple comparisons as our study was based on a priori hypotheses. However, there was a number of results that would withstand the strict Bonferroni correction $(0.05 / 8=0.006$ for Illinois Bully Scale peer and self-reports, ADHD, impulsivity, Life History of Aggression, psychiatric interview, anxiety and depressiveness scores), e.g., the genotype effects 
on IBS Victim and Fight scores, maladaptive impulsivity at age 18, the prevalence of anxiety disorders, and G ×AUD effect on anxiety scores.

Aggressiveness and impulsivity are often related to substance use (e.g., Cho et al., 2014) that in our sample is highly represented by alcohol abuse. We did not find any difference between TPH2 $-703 \mathrm{G} / \mathrm{T}$ genotypes in the prevalence of alcohol use disorder. Instead, we found lower prevalence of anxiety disorders and lower scores of anxiety and depressiveness in the TT homozygotes, and exploring further we discovered that the lowest anxiety and depressiveness were reported by the TT homozygotes if they had a history of alcohol use disorder. The very small number of subjects involved prevents making any strong conclusions, but one could wonder about the potential mechanism behind this interaction. While alcohol can elicit a variety of subjectively pleasurable effects (Zeiger et al., 2012) long-term alcohol abuse increases anxiety and lowers mood (e.g., Falk et al., 2008). The acute anxiolytic and mood enhancing effect of alcohol results partly from increased serotonin (5-HT) turnover while chronic alcohol use is associated with decreased 5-HT turnover (Heinz et al., 2006; Lovinger, 1997;). Our rather young subjects were predominantly alcohol abusers, not dependent on alcohol, and one could speculate that in the subjects with two copies of the T-allele, suggestively linked to enhanced 5-HT synthesis (Lin et al., 2007), the emergence harmful effects of alcohol may be delayed.

The TPH2-703G/T genotype effects were clearly more prominent in males. Sex differences have been reported before by Armbruster et al. (2010) who found that male T-allele carriers exhibited stronger startle responses compared to male GG homozygotes while in young women this effect appeared to be reversed. Overall, aggressive traits are more pronounced in males for evolutionary reasons (Archer, 2004), but why this relates to the TPH2 genotype remains to be elucidated. Similarly, thought-provoking is what could be the disadvantages brought about by the T-allele as it is the less frequent allele despite of the large positive differences in mental health of the TT homozygotes. The frequency of the T-allele in our sample (21.8\%) was similar to other Caucasian non-patient samples ranging from $18 \%$ to $23 \%$ (Gutknecht et al., 2007; Zhou et al., 2005). Are the TT homozygotes too "nice", lacking aggressiveness-related assertiveness? It is however possible that the favourable trait profile fades away with aging: Age dependent effects were reported by Armbruster et al. (2010) who found that genotype differences in startle responses emerged only in the sample of young adults, not in children or older adults. Cohort-specific effects have previously been reported: Reuter et al. (2007b) found that in their older cohort, females GG homozygotes 
started smoking earlier while in the younger cohort, smoking was initiated earlier by males GG homozygotes. While our two birth cohorts have revealed the existence of significant genotype by birth cohort interactions relevant to serotonergic neurotransmission (Vaht et al., 2015, 2014), the associations of aggressiveness and other traits with the $T P H 2-703 \mathrm{G} / \mathrm{T}$ genotype appeared similar in the two independent cohorts.

In conclusion, a low aggression and anxiety profile was found in the homozygotes for the minor T-allele of the TPH2 rs4570625. This is in line with the notion that higher serotonergic function inhibits aggressiveness and reduces anxiety, but indirectly suggests that at the level tryptophan hydroxylase genotype single alleles may have limited effect.

\section{Acknowledgements}

This work was supported by Estonian Ministry of Education and Science Projects IUT20-40 and IUT42-2, the EC FP7 Project Aggressotype (FP7-Health-2013-Innovation-1 602805) and the EC Horizon 2020 project CoCA (H2020-PHC-2015-667302). We are grateful to the ECPBHS study participants, their parents and the whole ECPBHS Team.

\section{References}

Af Klinteberg, B., Oreland, L., 1995. Hyperactive and aggressive behaviors in childhood as related to low platelet monoamine oxidase (MAO) activity at adult age: a longitudinal study of male subjects. Personal. Indiv. Differ. 19, 373-383.

Archer, J., 2004. Sex differences in aggression in real-world settings: a meta-analytic review. Rev. Gen. Psychol. 8 (4), 291-332.

Armbruster, D., Mueller, A., Strobel, A., Kirschbaum, C., Lesch, K.P., Brocke, B., 2010. Influence of functional tryptophan hydroxylase 2 gene variation and sex on the startle response in children, young adults, and older adults. Biol. Psychol. 83, 214-221.

Baehne, C.G., Ehlis, A.C., Plichta, M.M., Conzelmann, A., Pauli, P., Jacob, C., Gutknecht, L., Lesch, K.P., Fallgatter, A.J., 2009. Tph2 gene variants modulate response control processes in adult ADHD patients and healthy individuals. Mol. Psychiatry 14, 1032-1039.

Bortolato, M., Pivac, N., Muck Seler, D., Nikolac Perkovic, M., Pessia, M., Di Giovanni, G., 2013. The role of the serotonergic system at the interface of aggression and suicide. Neuroscience 236, $160-185$. 
Brown, S.M., Peet, E., Manuck, S.B., Williamson, D.E., Dahl, R.E., Ferrell, R.E., Hariri, A.R., 2005. A regulatory variant of the human tryptophan hydroxylase-2 gene biases amygdala reactivity. Mol. Psychiatry 10, 884-888.

Canli, T., Congdon, E., Gutknecht, L., Constable, R.T., Lesch, K.P., 2005. Amygdala responsiveness is modulated by tryptophan hydroxylase-2 gene variation. J. Neural Transm. 112, $1479-1485$.

Canli, T., Congdon, E., Todd, C.R., Lesch, K.P., 2008. Additive effects of serotonin transporter and tryptophan hydroxylase-2 gene variation on neural correlates of affective processing. Biol. Psychol. 79, 118-125.

Carkaci-Salli, N., Salli, U., Kuntz-Melcavage, K.L., Pennock, M.M., Ozgen, H., Tekin, I., Freeman, W.M., Vrana, K.E., 2011. TPH2 in the ventral tegmental area of the male rat brain. Brain Res. Bull. 84 (6), 376-380.

Chen, G.L., Vallender, E.J., Miller, G.M., 2008. Functional characterization of the human TPH2 5 ' regulatory region: untranslated region and polymorphisms modulate gene expression in vitro. Hum. Genet. 122, 645-657.

Cho, S.B., Heron, J., Aliev, F., Salvatore, J.E., Lewis, G., Macleod, J., Hickman, M., Maughan, B., Kendler, K.S., Dick, D.M., 2014. Directional relationships between alcohol use and antisocial behavior across adolescence. Alcohol. Clin. Exp. Res. 38 (7), 2024-2033.

Coccaro, E.F., Berman, M.E., Kavoussi, R.J., 1997. Assessment of life history of aggression: development and psychometric characteristics. Psychiatry Res. 73 (3), 147-157.

Espelage, D.L., Holt, M., 2001. Bullying and victimization during early adolescence: peer influences and psychosocial correlates. J. Emot. Abuse 2, 123-142.

Falk, D.E., Yi, H., Hilton, M.E., 2008. Age of onset and temporal sequencing of lifetime DSM-IV alcohol use disorders relative to comorbid mood and anxiety disorders. Drug Alcohol. Depend. 94, 234-245.

Gao, J., Pan, Z., Jiao, Z., Li, F., Zhao, G., Wei, Q., Pan, F., Evangelou, E., 2012. TPH2 gene polymorphisms and major depression - a meta-analysis. PLoS One 7, e36721.

Gutknecht, L., Jacob, C., Strobel, A., Kriegebaum, C., Müller, J., Zeng, Y., Markert, C., Escher, A., Wendland, J., Reif, A., Mössner, R., Gross, C., Brocke, B., Lesch, K.P., 2007. Tryptophan hydroxylase-2 gene variation influences personality traits and disorders related to emotional dysregulation. Int. J. Neuropsychopharmacol. 10, 309-320.

Harro, M., Eensoo, D., Kiive, E., Merenäkk, L., Alep, J., Oreland, L., Harro, J., 2001. Platelet monoamine oxidase in healthy 9- and 15-years old children: the effect of gender, smoking and puberty. Prog. Neuropsychopharmacol. Biol. Psychiatry 25, 1497-1511. 
Heinz, A., Mann, K., Weinberger, D.R., Goldman, D., 2006. Serotonergic dysfunction, negative mood states, and response to alcohol. Alcohol. Clin. Exp. Res. 25, 487-495.

Herrmann, M.J., Huter, T., Muller, F., Muhlberger, A., Pauli, P., Reif, A., Renner, T., Canli, T., Fallgatter, A.J., Lesch, K.P., 2007. Additive effects of serotonin transporter and tryptophan hydroxylase-2 gene variation on emotional processing. Cereb. Cortex 17, 1160-1163.

Kiive, E., Kurrikoff, T., Mäestu, J., Harro, J., 2010. Effect of a2A-adrenoceptor C-1291G genotype and maltreatment on hyperactivity and inattention in adolescents. Prog. Neuropsychopharmacol. Biol. Psychiatry 34, 219-224.

Kriegebaum, C., Song, N.N., Gutknecht, L., Huang, Y., Schmitt, A., Reif, A., Ding, Y.Q., Lesch, K.P., 2010. Brain-specific conditional and time-specific inducible Tph2 knockout mice possess normal serotonergic gene expression in the absence of serotonin during adult life. Neurochem. Int. $57(5), 512-517$.

Laas, K., Reif, A., Herterich, S., Eensoo, D., Lesch, K.P., Harro, J., 2010. The effect of a functional NOS1 promoter polymorphism on impulsivity is moderated by platelet MAO activity. Psychopharmacology 209, 255-261.

Lesch, K.P., Araragi, N., Waider, J., van den Hove, D., Gutknecht, L., 2012. Targeting brain serotonin synthesis: insights into neurodevelopmental disorders with longterm outcomes related to negative emotionality, aggression and antisocial behaviour. Philos. Trans. R. Soc. B. 367, 24262443.

Lehto, K., Vaht, M., Mäestu, J., Veidebaum, T., Harro, J., 2015. Effect of tryptophan hydroxylase2 gene polymorphism G-703 $\mathrm{T}$ on personality in a population representative sample. Prog. Neuropsychopharmacol. Biol. Psychiatry 57, 31-35.

Leys, C., Schumann, S., 2010. A nonparametric method to analyze interactions: the adjusted rank transform test. J. Exp. Soc. Psychol. 46 (4), 684-688.

Lin, Y.M., Chao, S.C., Chen, T.M., Lai, T.J., Chen, J.S., Sun, H.S., 2007. Association of functional polymorphisms of the human tryptophan hydroxylase 2 gene with risk for bipolar disorder in Han Chinese. Arch. Gen. Psychiatry 64, 1015-1024.

Lovinger, D.M., 1997. Serotonin's role in alcohol's effects on the brain. Neurotransmitter. Review 21, 114-120.

Mandelli, L., Antypa, N., Nearchou, F.A., Vaiopoulos, C., Stefanis, C.N., Serretti, A., Stefanis, N.C., 2012. The role of serotonergic genes and environmental stress on the development of depressive symptoms and neuroticism. J. Affect. Disord. 142, 82-89.

Miczek, K.A., DeBold, J.F., Hwa, L.S., Newman, E.L., de Almeida, R.M., 2015. Alcohol and violence: neuropeptidergic modulation of monoamine systems. Ann. N. Y. Acad. Sci. 1349, 96118. 
Mössner, R., Walitza, S., Geller, F., Scherag, A., Gutknecht, L., Jacob, C., Bogusch, L., Remschmidt, H., Simons, M., Herpertz-Dahlmann, B., Fleischaker, C., Schulz, E., Warnke, A., Hinney, A., Wewetzer, C., Lesch, K.P., 2006. Transmission disequilibrium of polymorphic variants in the tryptophan hydroxylase- 2 gene in children and adolescents with obsessivecompulsive disorder. Int. J. Neuropsychopharmacol. 9, 437-442.

Paaver, M., Kurrikoff, T., Nordquist, N., Oreland, L., Harro, J., 2008. The effect of 5-HTT gene promoter polymorphism on impulsivity depends on family relations in girls. Prog. Neuropsychopharmacol. Biol. Psychiatry 32, 1263-1268.

Perez-Rodriguez, M.M., Weinstein, S., New, A.S., Bevilacqua, L., Yuan, Q., Zhou, Z., Hodgkinson, C., Goodman, M., Koenigsberg, H.W., Goldman, D., Siever, L.J., 2010. Tryptophanhydroxylase 2 haplotype association with borderline personality disorder and aggression in a sample of patients with personality disorders and healthy controls. J. Psychiatr. Res. 44, 10751081.

Reuter, M., Kuepper, Y., Hennig, J., 2007a. Association between a polymorphism in the promoter region of the TPH2 gene and the personality trait of harm avoidance. Int. J. Neuropsychopharmacol. 10, 401-404.

Reuter, M., Hennig, J., Amelang, M., Montag, C., Korkut, T., Hueweler, A., Stürmer, T., $2007 b$. The role of the TPH1 and TPH2 genes for nicotine dependence: a genetic association study in two different age cohorts. Neuropsychobiology 56, 47-54.

Scheuch, K., Lautenschlager, M., Grohmann, M., Stahlberg, S., Kirchheiner, J., Zill, P., Heinz, A., Walther, D.J., Priller, J., 2007. Characterization of a functional promoter polymorphism of the human tryptophan hydroxylase 2 gene in serotonergic raphe neurons. Biol. Psychiatry 62, 12881294.

Sheehan, D.V., Lecrubier, Y., Sheehan, K.H., Amorim, P., Janavs, J., Weiller, E., Hergueta, E., Baker, T., Dunbar, G.C., 1998. The Mini International Neuropsychiatric Interview (M.I.N.I.): the development and validation of a structured diagnostic psychiatric interview for DSM-VI and ICD10. J. Clin. Psychiatry 59, 22-33.

Shlik, J., Aluoja, A., Kihl, E., 1999. MINI 5.0.0. Mini rahvusvaheline neuropsühhiaatriline intervjuu DSM -IV. Estonian version of MINI international neuropsychiatric interview.

Tomson, K., Merenäkk, L., Loit, H.M., Mäestu, J., Harro, J., 2011;al.. The relationship between serotonin transporter gene promoter polymorphism and serum lipid levels at young age in a longitudinal population-representative study. Prog. Neuropsychopharmacol. Biol. Psychiatry 35, 1857-1862.

Vaht, M., Kiive, E., Veidebaum, T., Harro, J., 2015;al.. A functional vesicular monoamine transporter 1 (VMAT1) gene variant is associated with affect and the prevalence of anxiety, affective, and alcohol use disorders in a longitudinal populationrepresentative birth cohort study. Int. J. Neuropsychopharmacol. 19 (7), 1-9. 
Vaht, M., Merenäkk, L., Mäestu, J., Veidebaum, T., Harro, J., 2014;al.. Serotonin transporter gene promoter polymorphism (5-HTTLPR) and alcohol use in general population: interaction effect with birth cohort. Psychopharmacology 231, 2587-2594.

Waider, J., Araragi, N., Gutknecht, L., Lesch, K.P., 2011. Tryptophan hydroxylase-2 (TPH2) in disorders of cognitive control and emotion regulation: a perspective. Psychoneuroendocrinology $36,393-405$.

Walitza, S., Renner, T.J., Dempfle, A., Konrad, K., Wewetzer, C., Halbach, A., HerpertzDahlmann, B., Remschmidt, H., Smidt, J., Linder, M., Flieri, L., Knölker, U., Friedel, S., Schäfer, H., Gross, C., Hebebrand, J., Warnke, A., Lesch, K.P., 2005. Transmission disequilibrium of polymorphic variants in the tryptophan hydroxylase-2 gene in attention-deficit/hyperactivity disorder. Mol. Psychiatry 10, 1126-1132.

Walther, D.J., Peter, J.U., Bashammakh, S., Hörtnagl, H., Voits, M., Fink, H., Bader, M., 2003. Synthesis of serotonin by a second tryptophan hydroxylase isoform. Science 299 (5603), 76.

Yoon, H.K., Kim, Y.K., 2009. TPH2-703G/T SNP may have important effect on susceptibility to suicidal behavior in major depression. Prog. Neuropsychopharmacol. Biol. Psychiatry 33 (3), 403409.

Zeiger, J.S., Haberstick, B.C., Corley, R.P., Ehringer, M.A., Crowley, T.J., Hewitt, J.K., Hopfer, C.J., Stallings, M.C., Young, S.E., Hyn Rhee, S., 2012. Subjective effects for alcohol, tobacco, and marijuana association with cross-drug outcomes. Drug. Alcohol Depend. 123, S52-S58.

Zhou, Z., Roy, A., Lipsky, R., Kuchipudi, K., Zhu, G., Taubman, J., Enoch, M.A., Virkkunen, M., Goldman, D., 2005. Haplotype-based linkage of tryptophan hydroxylase 2 to suicide attempt, major depression, and cerebrospinal fluid 5-hydroxyindoleacetic acid in 4 populations. Arch. Gen. Psychiatry 62, 1109-1118. 